\title{
Adenosine Mapping for Adenosine-Dependent Accessory Pathway Ablation
}

MARTIN J. LAPAGE, M.D., M.S., * MICHAEL J. WALSH, M.D.,† JOHN H. REED, M.D., M.P.H.,‡ and J. PHILIP SAUL, M.D.§

From the *Department of Pediatrics and Communicable Diseases, University of Michigan, Ann Arbor, Michigan; tDepartment of Pediatrics, Wake Forest University, Winston-Salem, North Carolina; ¥Department of Pediatrics, Children's Hospital of the King's Daughters, Norfolk, Virginia; and §Department of Pediatrics, Medical University of South Carolina, Charleston, South Carolina

Background: This study describes the use of adenosine during ablation procedures to allow conduction through adenosine-dependent accessory pathways (APS), which are inactive at the time of the procedure. The technique allows for successful mapping and ablation of these pathways.

Methods: Retrospective review of all patients undergoing AP ablation from 1998 to 2008 to identify patients with absent or intermittent AP conduction during electrophysiology study. Adenosine boluses were used to activate the AP for the purpose of mapping in each case.

Results: Adenosine mapping was utilized in seven patients. One patient had a concealed AP at baseline and six patients had manifest preexcitation at baseline but lost AP conduction during the case. Alternative methods of enhancing AP conduction were attempted in five patients, but failed. Acute ablation results included: four patients with complete elimination of AP conduction, two patients with AP conduction only with adenosine, and one patient with return of baseline preexcitation. Patients with residual antegrade conduction had right superior (anterior) septal APs considered too close to the atrioventricular conduction system for safe ablation. At median follow-up of 2 years, all three patients with residual AP conduction had recurrence of supraventricular tachycardia; the remainder remained free of preexcitation and SVT.

Conclusion: Adenosine mapping is a useful technique for facilitating AP ablation when pathway conduction is absent or inconsistent, and may enhance conduction in mechanically inhibited or previously ablated APs. Recurrence of AP conduction only during adenosine administration is predictive of late recurrence. (PACE 2014; 37:610-615)

adenosine, mapping, accessory pathway

\section{Introduction}

For a variety of reasons, accessory pathways (APs) that have been proven to cause symptomatic tachycardia may have intermittent or absent conduction during ablation procedures, making mapping and successful ablation considerably more difficult. These reasons include spontaneous loss of activation during sedation or anesthesia, reversible mechanical injury, and ablation energy application near the AP without permanent injury. Various techniques can be used to enhance AP conduction, including selective site pacing, Hisrefractory ventricular extrastimuli, and pharmacologic manipulation, but AP conduction may still remain elusive, frustrating the attempt at ablation.

Adenosine is a safe and useful tool in the electrophysiology lab. ${ }^{1}$ During preablation testing,

Address for reprints: Martin J. LaPage, M.D., University of Michigan, 1540 E. Hospital Drive, Ann Arbor, MI 48109. Fax: 734-936-9470; e-mail: mlapage@med.umich.edu

Received August 15, 2013; revised October 2, 2013; accepted November 3, 2013.

doi: $10.1111 /$ pace. 12324 it can be used to reveal latent preexcitation, ${ }^{2}$ in which antegrade AP conduction is concealed due to earlier ventricular activation via the AV node. Although retrograde AP conduction may be blocked by adenosine in occasional $\mathrm{APs},{ }^{3}$ adenosine can be used postablation to help confirm successful AP ablation ${ }^{4}$ by demonstrating that all atrioventricular (AV) and ventriculoatrial (VA) conduction blocks with adenosine.

Recently, the use of adenosine to unmask transient residual conduction after pulmonary vein isolation for atrial fibrillation has been the subject of multiple studies, showing improvement in ablation outcomes when it is used for this purpose. ${ }^{5}$ The concept of an adenosinedependent concealed AP has been the subject of a case report. ${ }^{6}$ This study describes our experience with adenosine-dependent APs and using "adenosine mapping" to successfully ablate these APs.

\section{Data Collection}

With the approval of our institutional review board, all AP ablation procedures at the MUSC Children's Hospital between 1998 and 2008 
were retrospectively reviewed to identify the use of adenosine administered specifically for the purposes of mapping. This did not include the use of adenosine for routine pre- or postablation diagnostic testing.

\section{Adenosine Mapping Technique}

After informed consent, standard electrophysiology study techniques were used. Most procedures were performed under general anesthesia using catheters placed in the coronary sinus, the right ventricle, and the His bundle region, in addition to a mapping and ablation catheter. Routine programmed stimulation of the atria and ventricle were performed. Left-sided APs were approached via transseptal catheterization. Use of radiofrequency or cryoablation was based on location of the AP.

AP mapping was performed using standard techniques to assess early and eccentric antegrade or retrograde conduction. If the AP could be localized in this manner, mapping and ablation were performed. However, in cases when AP conduction was intermittent or absent at either the initiation of, or during, the case, adenosine was administered as a $0.1-0.2 \mathrm{mg} / \mathrm{kg}$ per bolus (usual max dose $12 \mathrm{mg}$ ) given via a central sheath or catheter and followed by a 10-cc saline flush with the goal to revive AP conduction. When preexcitation or retrograde conduction through the AP occurred only during the effect of the adenosine bolus, mapping was performed only during this transient adenosine effect. For activation mapping of retrograde conduction, ventricular pacing could commence prior to adenosine administration. Examples of adenosinerevived AP conduction are shown in Figures 1 and 2. Repeated doses of adenosine were given and catheter manipulations were performed between doses. During the transient adenosine effect, local $\mathrm{V}: \mathrm{A}$ or $\mathrm{A}: \mathrm{V}$ times were measured. When earliest ventricular activation or shortest $\mathrm{V}$ :A time was identified, the location was ablated. There was no limit to the number of doses or the cumulative amount of adenosine given.

The cycle of mapping and ablation was continued until AP conduction remained absent. In some cases, where the AP was determined to be very close to the normal AV conduction tissue during adenosine mapping and the AP electrophysiological characteristics were low risk, complete elimination of AP conduction was not performed, as would be the case for a continuously activated AP. Postprocedure all patients were observed in the hospital for 6-24 hours, and an electrocardiogram performed prior to discharge.

\section{Results}

\section{Patients}

The above-described technique of adenosine mapping was used in seven electrophysiology studies from January 1998 through August 2008. Patient and electrophysiology study characteristics are shown in Table I. Six patients had manifest preexcitation at baseline, while one patient had a concealed AP and documented supraventricular tachycardia (SVT) after undergoing a prior ablation elsewhere for preexcitation and SVT.

\section{Use of Adenosine Mapping}

Adenosine mapping was utilized in three different situations: (1) absent or intermittent AP conduction at baseline, (2) mechanical trauma due to catheter manipulation eliminates conduction the AP, and (3) ablation eliminates AP conduction with return of conduction only during the transient effect of postablation adenosine testing. In five patients, additional methods for enhancing AP conduction were attempted without success, including esmolol to lower heart rate and slow AV nodal conduction, phenylephrine for reflex vagal activation and to lower heart rate, and isoproterenol. The patient-specific adenosine dosages are shown in Table I. No patient manifested adverse effects from the administration of adenosine.

\section{Ablation}

Radiofrequency energy was used for five patients and cryoablation for the remaining two patients due to AP proximity to the conduction system. At the completion of the procedures, four patients had complete elimination of AP conduction, two patients continued to have AP conduction only with adenosine, and one patient had return of baseline preexcitation with very lowrisk antegrade conduction properties. All three patients with residual antegrade conduction at the end of the procedure had right superior-septal APs considered to be too close to the atrioventricular conduction system to justify further attempts at ablation.

\section{Follow-Up}

Median follow-up was 2 years (range 0.25-8 years). All three patients with residual AP conduction (two only with adenosine) had recurrence of SVT. No other patient had recurrences of SVT or preexcitation.

\section{Discussion}

This study describes the specific use of adenosine to revive absent AP conduction for the purpose of mapping and demonstrates that 
LAPAGE, ET AL.






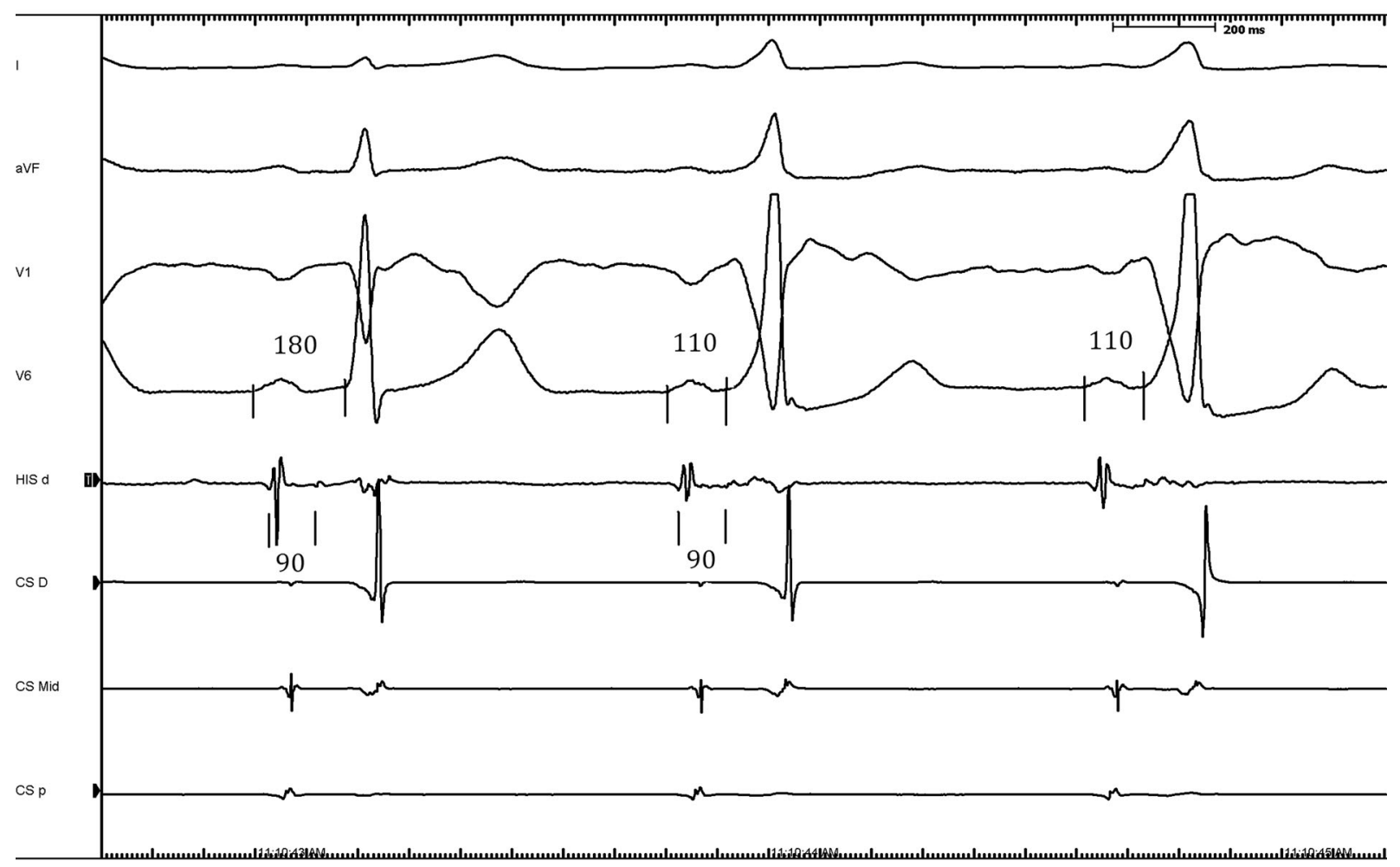

Figure 1. Intracardiac electrograms displayed from top to bottom: surface leads I, aVF, V1, and V6; His distal, CS distal to proximal. Measurements are in milliseconds. Vertical markings and notation on V6 show PR interval. Vertical markings and notation on His-d show AH interval. Preexcitation had been lost after mechanical trauma to the AP by the catheter. Here, a bolus of adenosine causes transient return of preexcitation. Note that when preexcitation appears the PR shortens, while the AH remains constant, indicating that the adenosine is inducing preexcitation and not simply revealing latent preexcitation by delaying the $A H$ conduction. $A H=$ atrial-His; $C S=$ coronary sinus.

successful ablation can be guided by the intermittent mapping possible during this transient adenosine effect. In this study, the use of adenosine for mapping an AP was critical in three situations: absent AP conduction at baseline, absent AP conduction due to mechanical injury, and absent AP conduction due to partially successful ablation. In these situations, adenosine mapping may be the only path to successful ablation.

Use of adenosine in the electrophysiology laboratory for diagnostic purposes prior to and after mapping and ablation ${ }^{2-4}$ has been previously published. The ability of adenosine to uncover intermittent conduction in APs has also been described, ${ }^{7-9}$ but only during intraoperative mapping or for diagnostic purposes. Recently, adenosine has been the subject of several studies on catheter ablation for atrial fibrillation in which it is used to revive conduction during pulmonary vein isolation. Ablation outcomes are improved when the transient adenosine-induced pulmonary venous reconnection is eliminated with further ablation lesions. Additionally, this effect of adenosine to revive dormant conduction across the cavo-tricuspid isthmus following atrial flutter ablation has been the subject of study. ${ }^{10}$

The mechanism by which adenosine allows conduction through the injured tissue is likely related to adenosine's hyperpolarizing effects on atrial myocardium, including AP tissue. ${ }^{1}$ Injury to myocardial tissue reduces the negative transmembrane potential, thereby inactivating $\mathrm{Na}$ channels and causing phase 4 block. Adenosine's hyperpolarizing effects correct this hypopolarization, thereby reviving excitability and allowing for transient return of conduction during its effect. As an anectodal observation, the "reviving" effect of adenosine on the AP routinely occurred several beats after the onset of AV node block by the drug. Adenosine is known to have different affinities for receptors in different myocardial tissue ${ }^{1}$ and this property may explain the delay between the manifestation of effects.

Two patients continued to have only adenosine-inducible AP conduction at the case 


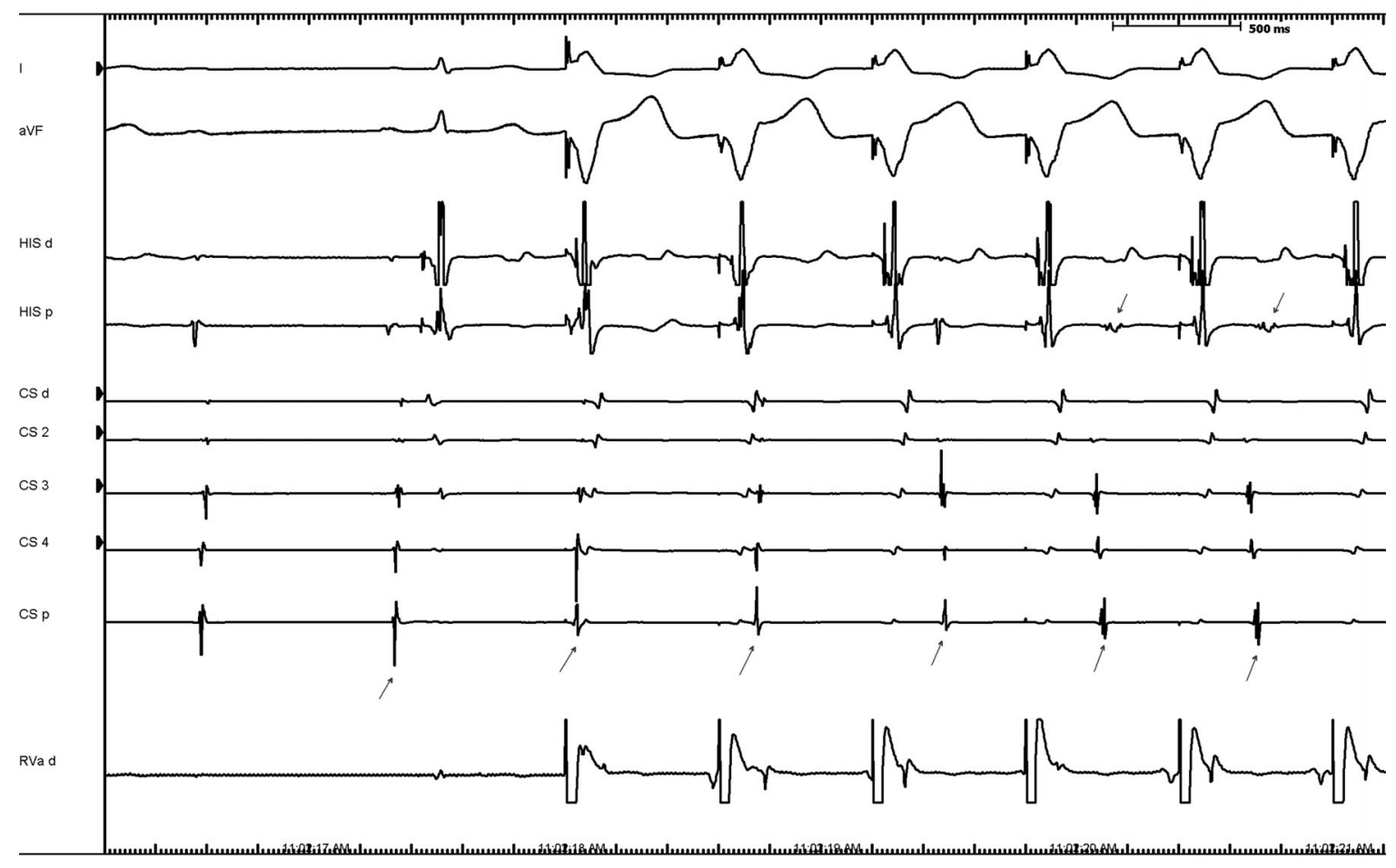

Figure 2. Intracardiac electrograms displayed from top to bottom: surface leads I and aVF, His distal and proximal, $C S$ distal to CS proximal, and RV apex. This patient had no evidence of antegrade AP conduction or retrograde conduction at baseline, including absent retrograde conduction during lateral LV wall pacing (not shown). She had undergone two prior ablation procedures for Wolff-Parkinson-White syndrome and had a documented recurrence of $S V T$ following the second ablation. The figure shows electrograms following adenosine administration and transient AV block; a single conducted sinus beat occurs prior to initiation of ventricular pacing. Eccentric retrograde activation of the atrium via a left lateral AP occurs with ventricular pacing. This is the first revelation of AP conduction during this electrophysiology study. $A P=$ accessory pathway; $A V=$ atrioventricular; $C S=$ coronary sinus; $L V=$ left ventricular; $R V=$ right ventricular; $S V T=$ supraventricular tachycardia.

conclusion. Further ablation attempts were deemed risky due to proximity to the His bundle. Notably, both of these patients had a subsequent recurrence of SVT, which may indicate that residual adenosine-inducible conduction at the case end is a marker for recurrence, similar to that described for pulmonary vein isolation ${ }^{5}$; however, the number of subjects is quite small. It is also important to note that none of our patients manifested adverse effects from adenosine and we found no data to suggest adverse effect from a high cumulative dose of adenosine. Notably, adenosine is known to cause rather noxious symptoms in awake patients and those under moderate ("conscious") sedation may experience these; however, the consequence of this could not be determined in this study as all cases were performed under general anesthesia. One indirect adverse effect of high adenosine utilization is the increased cost of the procedure. A 6-mg vial of adenosine incurs a charge of $\sim \$ 40$, which after
20 doses (40 vials) would add significantly to the overall cost of the procedure. However, given the observation that all three of the patients with residual conduction during adenosine mapping recurred, the cost of a second ablation at a future date imposes a far greater cost than the use of adenosine for mapping.

\section{Conclusion}

AP ablation may be complicated by absence of AP conduction due to tissue injury. Adenosine may transiently revive conduction through the injured AP due to its physiologic effects on atrial tissue. Adenosine mapping is a safe and useful technique in this circumstance and may be critical for absolute success. Return of AP conduction during adenosine administration may be predictive of late recurrence. Evaluation of $\mathrm{AV}$ and VA conduction after adenosine bolus should be routine after an apparently successful AP ablation. 


\section{ADENOSINE MAPPING FOR ACCESSORY PATHWAY ABLATION}

\section{References}

1. Lerman BB, Belardinelli L. Cardiac electrophysiology of adenosine. Basic and clinical concepts. Circulation 1991; 83:1499-1509.

2. Garratt CJ, Antoniou A, Griffith MJ, Ward DE, Camm, AJ. Use of intravenous adenosine in sinus rhythm as a diagnostic test for latent preexcitation. Am J Cardiol 1990; 65:868-873.

3. Fishberger SB, Saul JP, Triedman JK, Epstein MR, Walsh EP. Use of adenosine-sensitive nondecremental accessory pathways in assessing the results of radiofrequency catheter ablation. Am J Cardiol 1995; 75:1278-1281.

4. Walker KW, Silka MJ, Haupt D, Kron J, McAnulty JH, Halperin BD. Use of adenosine to identify patients at risk for recurrence of accessory pathway conduction after initially successful radiofrequency catheter ablation. Pacing Clin Electrophysiol 1995; 18(3 Pt 1):441446.

5. McLellan AJ, Kumar S, Smith C, Morton JB, Kalman JM, Kistler $\mathrm{PM}$. The role of adenosine following pulmonary vein isolation in patients undergoing catheter ablation for atrial fibrillation: A systematic review. J Cardiovasc Electrophysiol 2013; 24:742-751.
6. Tint D, Kun C, Beke I, Csanadi Z. Adenosine-dependent concealed accessory pathway. Pacing Clin Electrophysiol 2011; 35:e91e93.

7. Ellenbogen KA, Rogers R, Damiano R. Utility of adenosine administration during intraoperative mapping in a patient with the Wolff-Parkinson-White syndrome. Pacing Clin Electrophysiol 1991; 14:985-988

8. Alegret JM, Vinolas X, Palazon O, Vernis JM, Ferrer A, Oter R. Intermittent pre-excitation after adenosine administration. Revista Espanola de Cardiologia 2000; 53:1132-1135.

9. Morgan-Hughes NJ, Griffith MJ, McComb JM. Intravenous adenosine reveals intermittent preexcitation by direct and indirect effects on accessory pathway conduction. Pacing Clin Electrophysiol 1993; 16:2098-2103.

10. Vijayaraman P, Dandamudi G, Naperkowski A, Oren J, Storm R, Ellenbogen KA. Adenosine facilitates dormant conduction across cavotricuspid isthmus following catheter ablation. Heart Rhythm 2012; 9:1785-1788. 